\title{
Multivariate $q$-Bernstein-Schurer-Kantorovich Operators
}

\author{
D. K. Vishwakarma ${ }^{1}$, Artee $^{1}$, Alok Kumar ${ }^{1}$ and Ajay Kumar ${ }^{2}$
}

1. Department of Computer Science, Dev Sanskriti Vishwavidyalaya, Haridwar-249411, Uttarakhand, India.

2. Department of Mathematics, Motilal Nehru National Institute of Technology, Allahabad-211004, U.P., India.

Received: March 05, 2016 / Accepted: April 02, 2016 / Published: June 25, 2016.

\begin{abstract}
The purpose of this paper is to construct a multivariate generalization of a new kind of Kantorovich type $q$-Bernstein-Schurer operators. First, we establish the moments of the operators and then prove the rate of convergence by using the modulus of continuity. Finally, we obtain the degree of approximation by means of Lipschitz type class.
\end{abstract}

Keywords: $q$-Bernstein-Schurer-Kantorovich operators, rate of convergence, modulus of continuity, Lipschitz type class, multivariate operators.

\section{Introduction}

In recent years, one of the most interesting area of research in approximation theory is the application of $q$-calculus. Lupas and Phillips [16] was the first person who initiated the $q$-type generalization of linear positive operators. He introduced the $q$-analogue of well known Bernstein polynomials and obtained the rate of convergence and Voronovskaja type theorem for these operators. After that $q$-parametric operators is an active area of research in the field of approximation theory.

In [13], Muraru constructed the $q$-Bernstein-Schurer operators defined by

$$
B_{n, p}(f ; x)=\sum_{k=0}^{n+p} b_{n+p, k}(q ; x) f\left(\frac{[k]_{q}}{[n]_{q}}\right), x \in[0,1]
$$

where $b_{n+p, k}(q ; x)=\left(\begin{array}{c}n+p \\ k\end{array}\right)_{q} x^{k}(1-x)_{q}^{n+p-k}$ and

$$
(1-x)_{q}^{m}=\left\{\begin{array}{cc}
1, & m=0, \\
(1-x)(1-q x) \ldots\left(1-q^{m-1} x\right), & m \in N
\end{array}\right.
$$

for $x \in[0,1]$ and $0<q<1$. For $p=0$, the

Corresponding author: D. K. Vishwakarma, Department of Computer Science, Dev Sanskriti Vishwavidyalaya, India. E-mail: dkvishwa007@gmail.com. sequence of operators (1) reduces to the $q$-Bernstein polynomials (see [16]).

Dalmanoglu [4] studied some approximation properties of Kantorovich type generalization of $q$-Bernstein operators. In [17], Radu has obtained the statistical convergence of $q$-Bernstein-Kantorovich polynomials. Also, the Kantorovich type generalizations of the linear positive operators based on $q$-integers were studied by some authors (see [8], [10], [15], etc.). Recently, Agrawal, Finta and Sathish Kumar [1] introduced a new Kantorovich type generalization of the $q$-Bernstein-Schurer operators defined in [13] as follows

$$
\begin{aligned}
& K_{n, p}(f ; q, x) \\
& =[n+1]_{q} \sum_{k=0}^{n+p} b_{n+p, k}^{q}(x) q^{-k} \int_{[k]_{q} /[n+1]_{q}}^{[k+1]_{q} /[n+1]_{q}} f(t) d_{q}^{R} t, \\
& x \in[0,1] .
\end{aligned}
$$

In 2014, Same authors introduced Bivariate $q$ -Bernstein-Schurer operators defined as

$$
\begin{gathered}
K_{n_{1}, n_{2}, p}\left(f ; q_{n_{1}}, q_{n_{2}}, x, y\right) \\
=\left[n_{1}+1\right]_{q_{n_{1}}}\left[n_{2}+1\right]_{q_{n_{2}}} \sum_{k_{1}=0}^{n_{1}+p} \sum_{k_{2}=0}^{n_{2}+p} b_{n_{1}+p, n_{2}+p, k_{1}, k_{2}}^{q_{n_{1}}, q_{n_{2}}}(x, y) q_{n_{1}}^{-k_{1}} q_{n_{2}}^{-k_{2}}
\end{gathered}
$$


$\times \int_{\left[k_{2}\right]_{q_{n_{2}}} /\left[k_{2}+1\right]_{q_{n_{2}}}}^{\left[\left[k_{2}+1\right]_{q_{n_{2}}} /\left[n_{2}+1\right]_{q_{n_{2}}}\right.} \int_{\left[k_{1}\right]_{q_{n_{1}}} /\left[n_{1}+1\right]_{q_{n_{1}}}}^{\left[k_{n_{1}}+1\right]_{q_{n_{1}}} /\left[n_{1}+1\right]_{q_{1}}} f(t, s) d_{q_{n_{1}}}^{R}(t) d_{q_{n_{2}}}^{R}(s)$,

where $(x, y) \in[0,1] \times[0,1]$ and

$b_{n_{1}+p, n_{2}+p, k_{1}, k_{2}}^{q_{n_{1}}, q_{n_{2}}}(x, y)=$

$\left(\begin{array}{c}n_{1}+p \\ k_{1}\end{array}\right)_{q_{n_{1}}}\left(\begin{array}{c}n_{2}+p \\ k_{2}\end{array}\right)_{q_{n_{2}}} x^{k_{1}} y^{k_{2}}(1-x)_{q_{n_{1}}}^{n_{1}+p-k_{1}}(1-y)_{q_{n_{2}}}^{n_{2}+p-k_{2}}$.

Stancu [19], was the first person who introduced the linear positive operators in two and several variables. Later on, B $\breve{a}$ rbosu [3] considered the bivariate $q$-Bernstein polynomials and studied some Korovkin type approximation results. In [6], Erkuş and Duman proved the Korovkin-type approximation theorem for the bivariate linear positive operators to the functions in space $H_{\omega_{2}}$. Doğru and Gupta [5] studied the rate of convergence of the bivariate generalization of $q$-MKZ operators. In 2009, Ersan and Doğru [7] obtained the statistical approximation properties of $q$-Bleimann, Butzer and Hahn operators. Recently, Örkcui [14] constructed the bivariate generalization of the $q$-Sz $a$ sz-Mirakjan-Kantorovich operators by using the $q$-integral and obtained a weighted $A$-statistical convergence and the rate of pointwise approximation in terms of the modulus of continuity.
Now, we recall some basic definitions and notations of $q$-calculus [9]. For any fixed real number $q$ satisfying the conditions $0<q<1$, the $q$-integer $[k]_{q}$ and the $q$-factorial $[k]_{q} !$ for $k \in\{0,1,2 \ldots\}$ are defined as

$$
[k]_{q}=\left\{\begin{array}{cc}
\left(1-q^{k}\right)(1-q), & \text { if } q \neq 1 \\
k, & \text { if } q=1
\end{array}\right.
$$

and

$$
[k]_{q} !=\left\{\begin{array}{cc}
{[k]_{q}[k-1]_{q} \ldots[1]_{q},} & \text { if } k \geq 1 \\
1, & \text { if } k=0
\end{array}\right.
$$

respectively. For any integers $n, k$ satisfying $0 \leq k \leq n$, the $q$-binomial coefficient is given by

$$
\left(\begin{array}{l}
n \\
k
\end{array}\right)_{q}=\frac{[n]_{q} !}{[n-k]_{q} ![k]_{q} !} .
$$

Let $0 \leq a<b$. and $0<q<1$. Following [20], we consider the Riemann type $q$-integral is defined as

$$
\int_{a}^{b} f(t) d_{q}^{R} t=(1-q)(b-a) \sum_{i=0}^{\infty} f\left(a+(b-a) q^{i}\right) q^{i} .
$$

This Riemann type $q$-integral is appropriate to derive the $q$-analogues of some well-known integral inequalities. Then Riemann type $q$-integral for a multivariate function is given by

$$
\begin{gathered}
\int_{a_{m}}^{b_{m}} \int_{a_{m-1}}^{b_{m-1}} \ldots \int_{a_{1}}^{b_{1}} f\left(t_{1}, t_{2}, \ldots, t_{m}\right) d_{q_{1}}^{R} t_{1} d_{q_{2}}^{R} t_{2} \ldots d_{q_{m}}^{R} t_{m} \\
=\left(1-q_{1}\right)\left(1-q_{2}\right) \ldots\left(1-q_{m}\right)\left(b_{1}-a_{1}\right)\left(b_{2}-a_{2}\right) \ldots\left(b_{m}-a_{m}\right) \sum_{i_{m}=0}^{\infty} \ldots \sum_{i_{2}=0}^{\infty} \sum_{i_{1}=0}^{\infty} \\
f\left(a_{1}+\left(b_{1}-a_{1}\right) q_{1}{ }_{1}^{i_{1}}, a_{2}+\left(b_{2}-a_{2}\right) q_{2}{ }^{i_{2}}, \ldots, a_{m}+\left(b_{m}-a_{m}\right) q_{m}{ }^{i_{m}}\right) q_{1}^{i_{1}} q_{2}^{i_{2}} \ldots q_{m}{ }^{i_{m}} .
\end{gathered}
$$

where $0 \leq a_{i}<b_{i}$ and $0<q_{i}<1, i=1,2, \ldots, m$.

\section{Construction of Operators}

In this study, we construct a multivariate case of new Kantorovich type generalization of the $q$-Bernstein-Schurer operators defined in [13]. In what follows, let $I=[0,1+p], \quad$ where $p \in \mathbf{N}^{0}=\mathbf{N} \cup\{0\} \quad$ and $\quad J=[0,1] \quad$. For $I^{m}=I \times I \ldots \times I$, let $C\left(I^{m}\right)$ denote the space of all real valued continuous functions on $I^{m}$ endowed with the norm

$$
\begin{aligned}
& \|f\|_{I}=\sup _{\left(x_{1}, x_{2}, \ldots, x_{m}\right) \in I^{m}}\left|f\left(x_{1}, x_{2}, \ldots, x_{m}\right)\right| . \\
& \text { For } f \in C\left(I^{m}\right) \text { and } 0<q_{n_{i}}<1, i=1,2, \ldots, m,
\end{aligned}
$$
then we define the multivariate case of the Kantorovich type $q$-Bernstein-Schurer operators as follows: 


$$
\begin{aligned}
& K_{n_{1}, n_{2}, \ldots, n_{m}, p}\left(f ; q_{n_{1}}, q_{n_{2}}, \ldots, q_{n_{m}}, x_{1}, x_{2}, \ldots, x_{m}\right) \\
& =\left[n_{1}+1\right]_{q_{n_{1}}}\left[n_{2}+1\right]_{q_{n_{2}}} \ldots\left[n_{m}+1\right]_{q_{n_{m}}} \sum_{k_{1}=0}^{n_{1}+p} \sum_{k_{2}=0}^{n_{2}+p} \ldots \sum_{k_{m}=0}^{n_{m}+p} b_{n_{1}+p, n_{2}+p, \ldots, n_{m}+p, k_{1}, k_{2}, \ldots, k_{m}}^{q_{n_{1}}, q_{n_{2}}, \ldots, q_{n_{m}}}\left(x_{1}, x_{2}, \ldots, x_{m}\right) \\
& \times q_{n_{1}}^{-k_{1}} q_{n_{2}}^{-k_{2}} \cdots q_{n_{m}}^{-k_{m}} \int_{\left.\left[k_{m}\right]\right]_{q_{n_{m}}} /\left[n_{m}+1\right]_{q_{n_{m}}}}^{\left[k_{m}+1\right]_{n_{n_{m}}} /\left[n_{m}+1\right]_{q_{n_{m}}}} \int_{\left[k_{m-1}\right]_{q_{n_{m-1}}} /\left[n_{m-1}+1\right]_{q_{n_{m-1}}}}^{\left[k_{m-1}+1\right]_{q_{n_{m-1}}} /\left[n_{m-1}+1\right]_{q_{m-1}}} \ldots \\
& \int_{\left[k_{1}\right]_{q_{n_{1}}} /\left[n_{1}+1\right]_{q_{n_{1}}}}^{\left[k_{1}+1\right]_{q_{n_{1}}} /\left[n_{1}+1\right]_{q_{1}}} f\left(t_{1}, t_{2}, \ldots, t_{m}\right) d_{q_{n_{1}}}^{R} t_{1} d_{q_{n_{2}}}^{R} t_{2} \ldots d_{q_{n_{m}}}^{R} t_{m},
\end{aligned}
$$

where

$$
\begin{gathered}
b_{n_{1}+p, n_{2}+p, \ldots, n_{m}+p, k_{1}, k_{2}, \ldots, k_{m}}^{q_{n_{1}}, q_{n_{n}}, \ldots, q_{n_{n}}}\left(x_{1}, x_{2}, \ldots, x_{m}\right) \\
=\left(\begin{array}{c}
n_{1}+p \\
k_{1}
\end{array}\right)_{q_{n_{1}}}\left(\begin{array}{c}
n_{2}+p \\
k_{2}
\end{array}\right)_{q_{n_{2}}} \ldots\left(\begin{array}{c}
n_{m}+p \\
k_{m}
\end{array}\right)_{q_{n_{m}}} x_{1}{ }^{k_{1}} x_{2}{ }^{k_{2}} \ldots x_{m}{ }^{k_{m}} \\
\left(1-x_{1}\right)_{q_{n_{1}}}^{n_{1}+p-k_{1}}\left(1-x_{2}\right)_{q_{n_{2}}+p-k_{2}}^{n_{2}} \ldots\left(1-x_{m}\right)_{q_{n_{m}}+p-k_{m}}^{n_{n_{m}}} .
\end{gathered}
$$

The purpose of this study is to obtain some approximation properties of a Kantorovich type multivariate generalization of $q$-Bernstein-Schurer operators. We obtain the rate of convergecne by using the modulus of continuity and degree of approximation in terms of Lipschitz type space.

\section{Basic Results}

In what follows, for $i=1,2, \ldots, m$, let $\left(q_{n_{i}}\right)$ be a sequence in $(0,1)$ satisfying $\quad q_{n_{i}} \rightarrow 1$ and $q_{n_{i}}^{n_{i}} \rightarrow 0$ as $n \rightarrow \infty$.

\section{Lemma}

1. Let

$$
e_{i_{1} i_{2} \ldots i_{m}}\left(x_{1}, x_{2}, \ldots, x_{m}\right)=x_{1}{ }^{i_{1}} x_{2}{ }^{i_{2}} \ldots x_{m}{ }^{i_{m}} \text {, }
$$$$
\left(x_{1}, x_{2}, \ldots, x_{m}\right) \in I^{m}
$$

$\left(i_{1}, i_{2}, \ldots, i_{m}\right) \in N^{0} \times N^{0} \times \ldots \times N^{0}$

with

$i_{1}+i_{2}+\ldots+i_{m} \leq m$ be the m-dimensional test functions. Then the following equalities hold for the operators given by (4).

$$
\begin{gathered}
K_{n_{1}, n_{2}, \ldots, n_{m}, p}\left(e_{00 \ldots .} ; q_{n_{1}}, q_{n_{2}}, \ldots, q_{n_{m}}, x_{1}, x_{2}, \ldots, x_{m}\right)=1 ; \\
K_{n_{1}, n_{2}, ., n_{i},, n_{m}, p}\left(e_{00 . .1 .0} ; q_{n_{1}}, q_{n_{2}}, ., q_{n_{i}}, ., q_{n_{m}}, x_{1}, x_{2}, ., x_{i}, ., x_{m}\right)=\frac{\left[n_{i}+p\right]_{q_{n_{i}}}}{\left[n_{i}+1\right]_{q_{n_{i}}}} \frac{2 q_{n_{i}}}{[2]_{q_{n_{i}}}} x_{i} \\
+\frac{1}{[2]_{q_{n_{i}}}\left[n_{i}+1\right]_{q_{n_{i}}}} ; \\
K_{n_{1}, n_{2}, ., n_{i}, ., n_{m}, p}\left(e_{00 . .2 .0} ; q_{n_{1}}, q_{n_{2}}, ., q_{n_{i}}, ., q_{n_{m}}, x_{1}, x_{2}, ., x_{i}, ., x_{m}\right)=\frac{1}{\left[n_{i}+1\right]_{q_{n_{i}}}^{2}[3]_{q_{n_{i}}}} \\
+\frac{q_{n_{i}}\left(3+5 q_{n_{i}}+4 q_{n_{i}}{ }^{2}\right)}{[2]_{q_{n_{i}}}[3]_{q_{n_{i}}}} \frac{\left[n_{i}+p\right]_{q_{n_{i}}}}{\left[n_{i}+1\right]_{q_{n_{i}}}} x_{i}+\frac{q_{n_{i}}^{2}\left(1+q_{n_{i}}+4 q_{n_{i}}{ }^{2}\right)}{[2]_{q_{n_{i}}}[3]_{q_{n_{i}}}} \frac{\left[n_{i}+p\right]_{q_{n_{i}}}\left[n_{i}+p-1\right]_{q_{n_{i}}}}{\left[n_{i}+1\right]_{q_{n_{i}}}^{2}} x_{i}{ }^{2} .
\end{gathered}
$$

Proof. Using (3), we obtain,

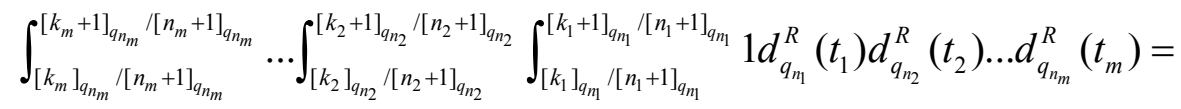

$$
\begin{aligned}
& \frac{q_{n_{1}}^{k_{1}} q_{n_{2}}^{k_{2}} \ldots q_{n_{m}}^{k_{m}}}{\left[n_{1}+1\right]_{q_{n_{1}}}\left[n_{2}+1\right]_{q_{n_{2}}} \ldots\left[n_{m}+1\right]_{q_{n_{m}}}} \text {. }
\end{aligned}
$$

Now, using the above equation in (4)and taking into account that $K_{n, p}(1 ; q, x)=1$ (see[[1], Lemma4 ]), we get 
Multivariate $q$-Bernstein-Schurer-Kantorovich Operators

237

$$
\begin{aligned}
& K_{n_{1}, n_{2}, \ldots, n_{m}, p}\left(e_{00 \ldots 0} ; q_{n_{1}}, q_{n_{2}}, \ldots, q_{n_{m}}, x_{1}, x_{2}, \ldots, x_{m}\right) \\
= & \sum_{k_{1}=0}^{n_{1}+p} \sum_{k_{2}=0}^{n_{2}+p} \ldots \sum_{k_{m}=0}^{n_{m}+p} b_{n_{1}+p, n_{2}+p, \ldots, n_{m}+p, k_{1}, k_{2}, \ldots, k_{m}}^{q_{n_{1},}, q_{n_{2}}, \ldots, q_{n_{m}}}\left(x_{1}, x_{2}, \ldots, x_{m}\right) \\
= & 1 .
\end{aligned}
$$

Now, applying (3) we have

$$
\begin{aligned}
& \int_{\left[k_{m}\right]_{q_{n_{m}}} /\left[n_{m}+1\right]_{q_{n_{m}}}}^{\left[k_{m}+1\right]_{q_{n_{m}}} /\left[n_{m}+1\right]_{q_{n_{m}}}} \ldots \int_{\left[k_{2}\right]_{q_{n_{2}}} /\left[n_{2}+1\right]_{q_{n_{2}}}}^{\left[k_{2}+1\right]_{q_{n_{2}}} /\left[n_{2}+1\right]_{q_{n_{2}}}} \int_{\left[k_{1}\right]_{q_{n_{1}}} /\left[n_{1}+1\right]_{q_{n_{1}}}}^{\left[k_{1}+1\right]_{q_{n_{1}}} /\left[n_{1}+1\right]_{q_{n_{1}}}} t_{i} d_{q_{n_{1}}}^{R}\left(t_{1}\right) d_{q_{n_{2}}}^{R}\left(t_{2}\right) \ldots d_{q_{n_{m}}}^{R}\left(t_{m}\right) \\
& =\frac{q_{n_{1}}^{k_{1}} \ldots q_{n_{i-1}}^{k_{i-1}}}{\left[n_{1}+1\right]_{q_{n_{1}}} \ldots\left[n_{i-1}+1\right]_{q_{n_{i-1}}}} \frac{q_{n_{i}}^{k_{i}}}{\left[n_{i}+1\right]_{q_{n_{i}}}}\left(\frac{\left[k_{i}\right]_{q_{n_{i}}}}{\left[n_{i}+1\right]_{q_{n_{i}}}}+\frac{q_{n_{i}}^{k_{i}}}{\left[n_{i}+1\right]_{q_{n_{i}}}[2]_{q_{n_{i}}}}\right) \frac{q_{n_{i+1}}^{k_{i+1}} \ldots q_{n_{m}}^{k_{m}}}{\left[n_{i+1}+1\right]_{q_{n_{i+1}}} \ldots\left[n_{m}+1\right]_{q_{n_{m}}}} .
\end{aligned}
$$

In view of (4) and

$$
\sum_{k_{i}=0}^{n_{i}+p} b_{n_{i}+p, k_{i}}^{q_{n_{i}}}\left(x_{i}\right) q_{n_{i}}^{k_{i}}=1-\left(1-q_{n_{i}}\right)\left[n_{i}+p\right]_{q_{n_{i}}} x_{i}
$$

we get

$$
\begin{aligned}
& K_{n_{1}, n_{2}, ., n_{i}, ., n_{m}, p}\left(e_{00.1 .0} ; q_{n_{1}}, q_{n_{2}}, ., q_{n_{i}}, ., q_{n_{m}}, x_{1}, x_{2}, ., x_{i}, ., x_{m}\right) \\
& =\sum_{k_{1}=0}^{n_{1}+p} \sum_{k_{2}=0}^{n_{2}+p} \ldots \sum_{k_{m}=0}^{n_{m}+p}\left(\begin{array}{c}
n_{1}+p \\
k_{1}
\end{array}\right)_{q_{n_{1}}}\left(\begin{array}{c}
n_{2}+p \\
k_{2}
\end{array}\right)_{q_{n_{2}}} \ldots\left(\begin{array}{c}
n_{m}+p \\
k_{m}
\end{array}\right)_{q_{n_{m}}} x_{1}^{k_{1}} x_{2}{ }^{k_{2}} \ldots x_{m}{ }^{k_{m}}\left(1-x_{1}\right)_{q_{n_{1}}}^{n_{1}+p-k_{1}} \\
& \times\left(1-x_{2}\right)_{q_{n_{2}}}^{n_{2}+p-k_{2}} \ldots\left(1-x_{m}\right)_{q_{n_{m}}}^{n_{m}+p-k_{m}}\left(\frac{\left[k_{i}\right]_{q_{n_{i}}}}{\left[n_{i}+1\right]_{q_{n_{i}}}}+\frac{q_{n_{i}}^{k_{i}}}{\left[n_{i}+1\right]_{q_{n_{i}}}[2]_{q_{n_{i}}}}\right) \\
& =\sum_{k_{i}=0}^{n_{i}+p}\left(\begin{array}{c}
n_{i}+p \\
k_{i}
\end{array}\right)_{q_{n_{i}}} x_{i}^{k_{i}}\left(1-x_{i}\right)_{q_{n_{i}}}^{n_{i}+p-k_{i}}\left(\frac{\left[k_{i}\right]_{q_{n_{i}}}}{\left[n_{i}+1\right]_{q_{n_{i}}}}+\frac{q_{n_{i}}^{k_{i}}}{\left[n_{i}+1\right]_{q_{n_{i}}}[2]_{q_{n_{i}}}}\right) \\
& =\frac{1}{\left[n_{i}+1\right]_{q_{n_{i}}}} \sum_{k_{i}=0}^{n_{i}+p}\left(\begin{array}{c}
n_{i}+p \\
k_{i}
\end{array}\right)_{q_{n_{i}}} x_{i}^{k_{i}}\left(1-x_{i}\right)_{q_{n_{i}}}^{n_{i}+p-k_{i}}\left[k_{i}\right]_{q_{n_{i}}} \\
& +\frac{1}{\left[n_{i}+1\right]_{q_{n_{i}}}[2]_{q_{n_{i}}}} \sum_{k_{i}=0}^{n_{i}+p}\left(\begin{array}{c}
n_{i}+p \\
k_{i}
\end{array}\right)_{q_{n_{i}}} x_{i}^{k_{i}}\left(1-x_{i}\right)_{q_{n_{i}}}^{n_{i}+p-k_{i}} q_{n_{i}}^{k_{i}}
\end{aligned}
$$

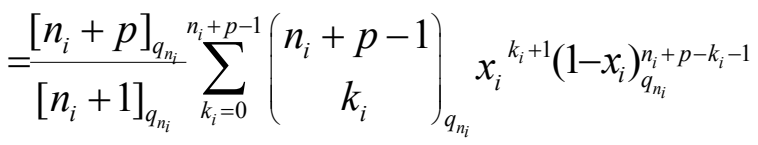

$$
\begin{aligned}
& +\frac{1}{\left[n_{i}+1\right]_{q_{n_{i}}}[2]_{q_{n_{i}}}} \sum_{k_{i}=0}^{n_{i}+p}\left(\begin{array}{c}
n_{i}+p \\
k_{i}
\end{array}\right)_{q_{n_{i}}} x_{i}^{k_{i}}\left(1-x_{i}\right)_{q_{n_{i}}}^{n_{i}+p-k_{i}} q_{n_{i}}^{k_{i}} \\
& =\frac{\left[n_{i}+p\right]_{q_{n_{i}}}}{\left[n_{i}+1\right]_{q_{n_{i}}}} x_{i}+\frac{1}{[2]_{q_{n_{i}}}\left[n_{i}+1\right]_{q_{n_{i}}}}\left(1-\left(1-q_{n_{i}}\right)\left[n_{i}+p\right]_{q_{n_{i}}} x_{i}\right) \\
& =\frac{\left[n_{i}+p\right]_{q_{n_{i}}}}{\left[n_{i}+1\right]_{q_{n_{i}}}} \frac{2 q_{n_{i}}}{[2]_{q_{n_{i}}}} x_{i}+\frac{1}{[2]_{q_{n_{i}}}\left[n_{i}+1\right]_{q_{n_{i}}}} .
\end{aligned}
$$

Again using (3), we find 
238

Multivariate $q$-Bernstein-Schurer-Kantorovich Operators

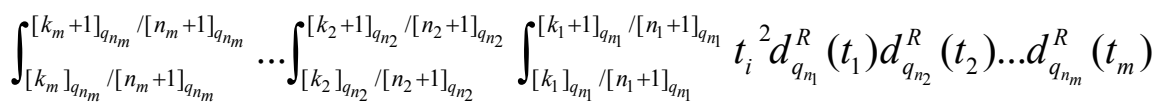

$$
\begin{aligned}
& =\frac{q_{n_{1}}^{k_{1}} \ldots q_{n_{i}}^{k_{i}}}{\left[n_{1}+1\right]_{q_{n_{1}}} \ldots\left[n_{i}+1\right]_{q_{n_{i}}}}\left(\frac{\left[k_{i}\right]_{q_{n_{i}}}^{2}}{\left[n_{i}+1\right]_{q_{n_{i}}}^{2}}+\frac{q_{n_{i}}^{2 k_{i}}}{\left[n_{i}+1\right]_{q_{n_{i}}}[3]_{q_{n_{i}}}}+\frac{2 q_{n_{i}}^{k_{i}}\left[k_{i}\right]_{q_{n_{i}}}}{\left[n_{i}+1\right]_{q_{n_{i}}}^{2}}\right) \\
& \times \frac{q_{n_{i+1}}^{k_{i+1}} \ldots q_{n_{m}}^{k_{m}}}{\left[n_{i+1}+1\right]_{q_{n_{i+1}}} \ldots\left[n_{m}+1\right]_{q_{n_{m}}}} .
\end{aligned}
$$

From (2),(5) and

$$
\sum_{k_{i}=0}^{n_{i}+p} b_{n_{i}+p, k_{i}}^{q_{n_{i}}}\left(x_{i}\right) q_{n_{i}}^{2 k_{i}}=1-\left(1-q_{n_{i}}^{2}\right)\left[n_{i}+p\right]_{q_{n_{i}}} x_{i}+q_{n_{i}}\left(1-q_{n_{i}}\right)^{2}\left[n_{i}+p\right]_{q_{n_{i}}}\left[n_{i}+p-1\right]_{q_{n_{i}}} x_{i}^{2},
$$

we get

$$
\begin{aligned}
& K_{n_{1}, n_{2},, n_{i},, n_{m}, p}\left(e_{00.2 .0} ; q_{n_{1}}, q_{n_{2}}, . ., q_{n_{i}}, ., q_{n_{m}}, x_{1}, x_{2}, ., x_{i}, ., x_{m}\right) \\
& =\sum_{k_{1}=0}^{n_{1}+p} \sum_{k_{2}=0}^{n_{2}+p} \ldots \sum_{k_{2}=0}^{n_{2}+p}\left(\begin{array}{c}
n_{1}+p \\
k_{1}
\end{array}\right)_{q_{m_{1}}}\left(\begin{array}{c}
n_{2}+p \\
k_{2}
\end{array}\right)_{q_{n_{2}}} \ldots\left(\begin{array}{c}
n_{m}+p \\
k_{m}
\end{array}\right)_{q_{n_{m}}} x_{1}{ }^{k_{1}} x_{2}{ }^{k_{2}} \ldots x_{m}{ }^{k_{m}}\left(1-x_{1}\right)_{q_{n_{1}}}^{n_{1}+p-k_{1}}
\end{aligned}
$$

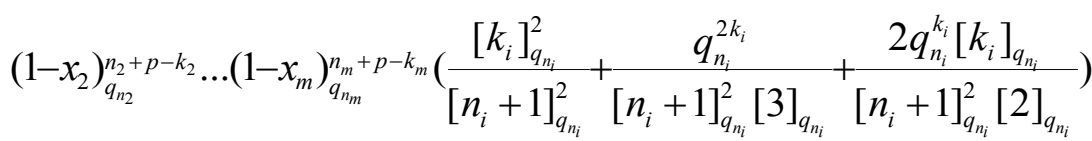

$$
\begin{aligned}
& =\sum_{k_{i}=0}^{n_{i}+p}\left(\begin{array}{c}
n_{i}+p \\
k_{i}
\end{array}\right)_{q_{n_{i}}} x_{i}^{k_{i}}\left(1-x_{i}\right)_{q_{n_{i}}}^{n_{i}+p-k_{i}}
\end{aligned}
$$

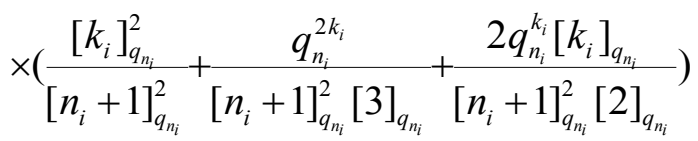

$$
\begin{aligned}
& =\sum_{k_{i}=0}^{n_{i}+p}\left(\begin{array}{c}
n_{i}+p \\
k_{i}
\end{array}\right)_{q_{n_{i}}} x_{i}^{k_{i}}\left(1-x_{i}\right)_{q_{n_{i}}}^{n_{i}+p-k_{i}} \frac{\left[k_{i}\right]_{q_{n_{i}}}^{2}}{\left[n_{i}+1\right]_{q_{n_{i}}}^{2}}+\sum_{k_{i}=0}^{n_{i}+p}\left(\begin{array}{c}
n_{i}+p \\
k_{i}
\end{array}\right)_{q_{n_{1}}} x_{i}{ }^{k_{i}}\left(1-x_{i}\right)_{q_{n_{i}}}^{n_{i}+p-k_{i}} \\
& \frac{2 q_{n_{i}}^{k_{i}}\left[k_{i}\right]_{q_{n_{i}}}}{\left[n_{i}+1\right]_{q_{n_{i}}}^{2}[2]_{q_{n_{i}}}}+\sum_{k_{i}=0}^{n_{i}+p}\left(\begin{array}{c}
n_{i}+p \\
k_{i}
\end{array}\right)_{q_{n_{i}}} x_{i}^{k_{i}}\left(1-x_{i}\right)_{q_{n_{i}}+p-k_{i}}^{n_{i}} \frac{q_{n_{i}}^{2 k_{i}}}{\left[n_{i}+1\right]_{q_{n_{i}}}^{2}[3]_{q_{n_{i}}}} \\
& =\frac{\left[n_{i}+p\right]_{q_{n_{i}}}}{\left[n_{i}+1\right]_{q_{n_{i}}}}\left(\frac{1}{\left[n_{i}+1\right]_{q_{n_{i}}}}+\frac{q_{n_{i}} x_{i}\left[n_{i}+p-1\right]_{q_{n_{i}}}}{\left[n_{i}+1\right]_{q_{n_{i}}}}\right) \\
& +\frac{2\left[n_{i}+p\right]_{q_{n_{i}}}\left[n_{i}+1\right]_{q_{n_{i}}}}{\left[n_{i}+1\right]_{q_{n_{i}}}^{2}[2]_{q_{n_{i}}}} x_{i}\left(1-\left(1-q_{n_{i}}\right)\left[n_{i}+p-1\right]_{q_{n_{i}}} x_{i}\right) \\
& +\frac{1}{\left[n_{i}+1\right]_{q_{n_{i}}}^{2}[3]_{q_{n_{i}}}}\left(1-\left(1-q_{n_{i}}^{2}\right)\left[n_{i}+p\right]_{q_{n_{i}}} x_{i}+q_{n_{i}}\left(1-q_{n_{1}}\right)^{2}\left[n_{i}+p\right]_{q_{n_{i}}}\left[n_{i}+p-1\right]_{q_{n_{i}}} x_{i}^{2}\right) \\
& =\frac{1}{\left[n_{i}+1\right]_{q_{n_{i}}}^{2}[3]_{q_{n_{i}}}}+\frac{q_{n_{i}}\left(3+5 q_{n_{i}}+4 q_{n_{i}}{ }^{2}\right)}{[2]_{q_{n_{i}}}[3]_{q_{n_{i}}}} \frac{\left[n_{i}+p\right]_{q_{n_{i}}}}{\left[n_{i}+1\right]_{q_{n_{i}}}^{2}} x_{i} \\
& +\frac{q_{n_{i}}^{2}\left(1+q_{n_{i}}+4 q_{n_{i}}{ }^{2}\right)}{[2]_{q_{n_{i}}}[3]_{q_{n_{i}}}} \frac{\left[n_{i}+p\right]_{q_{n_{i}}}\left[n_{i}+p-1\right]_{q_{n_{i}}}}{\left[n_{i}+1\right]_{q_{n_{i}}}^{2}} x_{i} \text {. }
\end{aligned}
$$


Hence, the proof is completed.

Remark 1. From Lemma 1, we get

$$
\begin{gathered}
K_{n_{1}, n_{2}, \ldots, n_{m}, p}\left(\left(t_{i}-x_{i}\right) ; q_{n_{1}}, q_{n_{2}}, \ldots, q_{n_{m}}, x_{1}, x_{2}, \ldots, x_{m}\right)=\frac{1}{[2]_{q_{n_{i}}}\left[n_{i}+1\right]_{q_{n_{i}}}} \\
+x_{i}\left(\frac{\left[n_{i}+p\right]_{q_{n_{i}}}}{\left[n_{i}+1\right]_{q_{n_{i}}}} \frac{2 q_{n_{i}}}{\left[2 q_{q_{n_{i}}}\right.}-1\right) ; \\
K_{n_{1}, n_{2}, \ldots, n_{m}, p}\left(\left(t_{i}-x_{i}\right)^{2} ; q_{n_{1}}, q_{n_{2}}, \ldots, q_{n_{m}}, x_{1}, x_{2}, \ldots, x_{m}\right)=\left(\frac{q_{n_{i}}^{2}\left(1+q_{n_{i}}+4 q_{n_{i}}{ }^{2}\right)}{[2]_{q_{n_{i}}}[3]_{q_{n_{i}}}}\right. \\
\left.\times \frac{\left[n_{i}+p\right]_{q_{n_{i}}}\left[n_{i}+p-1\right]_{q_{n_{i}}}}{\left[n_{i}+1\right]_{q_{n_{i}}}^{2}}-\frac{\left[n_{i}+p\right]_{q_{n_{i}}}}{\left[n_{i}+1\right]_{q_{n_{i}}}} \frac{4 q_{n_{n_{i}}}}{[2]_{q_{n_{i}}}}+1\right) x_{i}{ }^{2}+\left(\frac{q_{n_{i}}\left(3+5 q_{n_{i}}+4 q_{n_{i}}{ }^{2}\right)}{[2]_{q_{n_{i}}}[3]_{q_{n_{i}}}} \frac{\left[n_{i}+p\right]_{q_{n_{i}}}}{\left[n_{i}+1\right]_{q_{n_{i}}}^{2}}-\frac{2}{\left[n_{i}+1\right]_{q_{n_{i}}}[2]_{q_{n_{i}}}}\right) x_{i}+\frac{1}{\left[n_{i}+1\right]_{q_{n_{i}}}^{2}[3]_{q_{n_{i}}}} ;
\end{gathered}
$$

\section{Main Results}

Theorem 1. For any $f \in C\left(I^{m}\right)$, we have

$$
\lim _{n_{1}, n_{2}, \ldots, n_{m} \rightarrow \infty}\left\|K_{n_{1}, n_{2}, \ldots, n_{m}, p}(f)-f\right\|_{I}=0 .
$$

Proof. To proof our theorem, we need some notions concerning $j$ th projection and Korovkin subset according to [2]. For locally compact Hausdorff spaces $X_{1}, X_{2}, \ldots, X_{m}$ we denote $p r_{j}$ : $X_{1} \times X_{2} \times \ldots \times X_{m} \rightarrow X_{j}(j=1,2, \ldots, m)$ the $j$ th projection which is defined by $p r_{j}=x_{j}$ for every $x=\left(x_{1}, x_{2}, \ldots, x_{m}\right) \in X_{1} \times X_{2} \times \ldots \times X_{m}$. Let $\mathrm{X}$ and
$\mathrm{Y}$ be two locally compact Hausdorff spaces and $T: C(X) \rightarrow C(Y)$ be a positive linear operator. A subset $\mathrm{H}$ of $\mathrm{C}(\mathrm{X})$ is called Korovkin subset for $\mathrm{T}$ with respect to positive linear operator if it Satisfies the following property: if $L_{i i \in I}^{\leq}$is an arbitrary net of of positive linear operators from $\mathrm{C}(\mathrm{X})$ into $\mathrm{C}(\mathrm{y})$ such that $\sup _{i \in I} \| L_{i \|<\infty}$ and if $\lim _{i \in I \leq} L_{i}(h)=T(h)$ for all $h \in H$, then $\lim _{i \in I \leq} L_{i}(f)=T(f)$ for every $f \in C(X)$.

For $f \in C\left(I^{m}\right)$, the modulus of continuity for multivariate case is defined as follows:

$$
\begin{aligned}
& \omega\left(f ; \delta_{1}, \delta_{2}, \ldots, \delta_{m}\right)=\sup \left\{\left|f\left(t_{1}, t_{2}, \ldots, t_{m}\right)-f\left(x_{1}, x_{2}, \ldots, x_{m}\right)\right|:\left(t_{1}, t_{2}, \ldots, t_{m}\right),\left(x_{1}, x_{2}, \ldots, x_{m}\right) \in I^{m}\right. \\
& \text { and } \left.\left|t_{i}-x_{i}\right| \leq \delta_{i}, \text { for } i=1,2, \ldots m\right\},
\end{aligned}
$$

where $\omega\left(f ; \delta_{1}, \delta_{2}, \ldots, \delta_{m}\right)$ satisfies the following properties:

$$
\begin{aligned}
& \omega\left(f ; \delta_{1}, \delta_{2}, \ldots, \delta_{m}\right) \rightarrow 0 \text { if } \delta_{i} \rightarrow 0 \text { for } \\
& i=1,2, \ldots, m ; \\
& \left|f\left(t_{1}, t_{2}, \ldots, t_{m}\right)-f\left(x_{1}, x_{2}, \ldots, x_{m}\right)\right| \leq \\
& \omega\left(f, \delta_{1}, \delta_{2}, \ldots, \delta_{m}\right)\left(1+\left|t_{1}-x_{2}\right| / \delta_{1}\right)\left(1+\left|t_{2}-x_{2}\right| / \delta_{2}\right) \\
& \ldots\left(1+\left|t_{m}-x_{m}\right| / \delta_{m}\right) .
\end{aligned}
$$

Now, we give the estimate of the rate of convergence of the multivariate operators defined in (4).
Theorem 2. Let $f \in C\left(I^{m}\right)$ and $0<q_{n_{i}}<1$ for $i=1,2, \ldots, m$. Then for all $\left(x_{1}, x_{2}, \ldots, x_{m}\right) \in J^{m}$, we have

$$
\begin{aligned}
& \mid K_{n_{1}, n_{2}, \ldots n_{m}, p}\left(f ; q_{n_{1}}, q_{n_{2}} \ldots q_{n_{m}}, x_{1}, x_{2}, \ldots, x_{m}\right) \\
& -f\left(x_{1}, x_{2}, \ldots, x_{m}\right) \mid \\
& \leq 4 \omega\left(f ; \sqrt{\delta_{n_{1}}\left(x_{1}\right)}, \sqrt{\delta_{n_{2}}\left(x_{2}\right)}, \ldots, \sqrt{\delta_{n_{m}}\left(x_{m}\right)}\right),
\end{aligned}
$$

where $\quad \delta_{n_{i}}\left(x_{i}\right)=K_{n_{i}, p}\left(\left(t_{i}-x_{i}\right)^{2} ; q_{n_{i}}, x_{i}\right) \quad$ for $i=1,2, \ldots, m$. 
Proof. Using linearity and positivity of the operator $K_{n_{1}, n_{2}, \ldots, n_{m}, p}\left(f ; q_{n_{1}}, q_{n_{2}}, \ldots, q_{n_{m}}, x_{1}, x_{2}, \ldots, x_{m}\right)$, we have

$$
\begin{aligned}
& \quad\left|K_{n_{1}, n_{2}, \ldots, n_{m}, p}\left(f ; q_{n_{1}}, q_{n_{2}}, \ldots, q_{n_{m}}, x_{1}, x_{2}, \ldots, x_{m}\right)-f\left(x_{1}, x_{2}, \ldots, x_{m}\right)\right| \\
& \leq K_{n_{1}, n_{2}, \ldots, n_{m}, p}\left(\left|f\left(t_{1}, t_{2}, \ldots, t_{m}\right)-f\left(x_{1}, x_{2}, \ldots, x_{m}\right)\right| ; q_{n_{1}}, q_{n_{2}}, \ldots, q_{n_{m}}, x_{1}, x_{2}, \ldots, x_{m} \mid\right) \\
& \leq \omega\left(f ; \sqrt{\delta_{n_{1}}\left(x_{1}\right)}, \sqrt{\delta_{n_{2}}\left(x_{2}\right)} \ldots \sqrt{\delta_{n_{m}}\left(x_{m}\right)}\right)\left(K_{n_{1}, p}\left(1 ; q_{n_{1}}, x_{1}\right)+\frac{1}{\sqrt{\delta_{n_{1}}(x)}} K_{n_{1}, p}\left(\left|t_{1}-x_{1}\right| ; q_{n_{1}}, x_{1}\right)\right) \\
& \times\left(K_{n_{2}, p}\left(1 ; q_{n_{2}}, x_{2}\right)+\frac{1}{\sqrt{\delta_{n_{2}}(y)}} K_{n_{2}, p}\left(\left|t_{2}-x_{2}\right| ; q_{n_{2}}, x_{2}\right)\right) \\
& \ldots \times\left(K_{n_{m}, p}\left(1 ; q_{n_{m}}, x_{m}\right)+\frac{1}{\sqrt{\delta_{n_{m}}\left(x_{m}\right)}} K_{n_{m}, p}\left(\left|t_{m}-x_{m}\right| ; q_{n_{m}}, x_{m}\right)\right) .
\end{aligned}
$$

Now, applying the Cauchy-Schwarz inequality we have

$$
K_{n_{i}, p}\left(\left|t_{i}-x_{i}\right| ; q_{n_{i}}, x_{i}\right) \leq K_{n_{i}, p}\left(\left(t_{i}-x_{i}\right)^{2} ; q_{n_{i}}, x_{i}\right)^{1 / 2}\left(K_{n_{i}, p}\left(1 ; q_{n_{i}}, x_{i}\right)^{1 / 2} \text { for } i=1,2, \ldots, m .\right.
$$

Using the above, we get the desired result.

\subsection{Degree of approximation}

Now, we study the degree of approximation for the multivariate operators (4) by means of the Lipschitz class.

For $0<\alpha_{i}<1$ and $i=1,2, \ldots, m$, we define the Lipschitz class $\operatorname{Lip}_{M}\left(\alpha_{1}, \alpha_{2}, \ldots, \alpha_{m}\right)$ for the multivariate case as follows:

$$
\begin{aligned}
& \left|f\left(t_{1}, t_{2}, \ldots, t_{m}\right)-f\left(x_{1}, x_{2}, \ldots, x_{m}\right)\right| \leq \\
& M\left|t_{1}-x_{1}\right|^{\alpha_{1}}\left|t_{2}-x_{2}\right|^{\alpha_{2}} \ldots\left|t_{m}-x_{m}\right|^{\alpha_{m}} .
\end{aligned}
$$

Theorem 3. Let $f \in \operatorname{Lip}_{M}\left(\alpha_{1}, \alpha_{2}, \ldots \alpha_{m},\right)$. Then, we have

$$
\begin{aligned}
& \mid K_{n_{1}, n_{2}, \ldots, n_{m}, p}\left(f ; q_{n_{1}}, q_{n_{2}}, \ldots, q_{n_{m}}, x_{1}, x_{2}, \ldots, x_{m}\right) \\
& -f\left(x_{1}, x_{2}, \ldots, x_{m}\right) \mid \\
& \quad \leq M \delta_{n_{1}}^{\alpha_{1} / 2}\left(x_{1}\right) \delta_{n_{2}}^{\alpha_{2} / 2}\left(x_{2}\right) \ldots \delta_{n_{m}}^{\alpha_{m} / 2}\left(x_{m}\right),
\end{aligned}
$$

where $\delta_{n_{i}}\left(x_{i}\right)$ for $i=1,2, \ldots, m$ are defined as in Theorem 2.

Proof. By our hypothesis, we may write

$$
\begin{aligned}
& \left|K_{n_{1}, n_{2}, \ldots, n_{m}, p}\left(f ; q_{n_{1}}, q_{n_{2}}, \ldots, q_{n_{m}}, x_{1}, x_{2}, \ldots, x_{m}\right)-f\left(x_{1}, x_{2}, \ldots, x_{m}\right)\right| \\
\leq & K_{n_{1}, n_{2}, \ldots, n_{m}, p}\left(\left|f\left(t_{1}, t_{2}, \ldots, t_{m}\right)-f\left(x_{1}, x_{2}, \ldots, x_{m}\right)\right| ; q_{n_{1}}, q_{n_{2}}, \ldots, q_{n_{m}}, x_{1}, x_{2}, \ldots, x_{m}\right) \\
\leq & M K_{n_{1}, n_{2}, \ldots, n_{m}, p}\left(\left|t_{1}-x_{1}\right|^{\alpha_{1}}\left|t_{2}-x_{2}\right|^{\alpha_{2}} \ldots\left|t_{m}-x_{m}\right|^{\alpha_{m}} ; q_{n_{1}}, q_{n_{2}}, \ldots, q_{n_{m}}, x_{1}, x_{2}, \ldots, x_{m}\right) \\
= & M K_{n_{1}, p}\left(\left|t_{1}-x_{1}\right|^{\alpha_{1}} ; q_{n_{1}}, x_{1}\right) K_{n_{2}, p}\left(\left|t_{2}-x_{2}\right|^{\alpha_{2}} ; q_{n_{2}}, x_{2}\right) \ldots K_{n_{m}, p}\left(\left|t_{m}-x_{m}\right|^{\alpha_{m}} ; q_{n_{m}}, x_{m}\right) .
\end{aligned}
$$

Now, using the Hölder's inequality with

$$
\begin{array}{llll}
u_{1}=2 / \alpha_{1}, \quad v_{1}=2 /\left(2-\alpha_{1}\right), \quad u_{2}=2 / \alpha_{2}, & & \leq M K_{n_{1}, p}\left(\left(t_{1}-x_{1}\right)^{2} ; q_{n_{1}}, x_{1}\right)^{\frac{\alpha_{1}}{2}} K_{n_{1}, p}\left(1 ; q_{n_{1}}, x_{1}\right)^{\frac{2-\alpha_{1}}{2}} \\
v_{2}=2 /\left(2-\alpha_{2}\right), \ldots, u_{m}=2 / \alpha_{m}, \quad v_{m}=2 /\left(2-\alpha_{m}\right), & & \times K_{n_{2}, p}\left(\left(t_{2}-x_{2}\right)^{2} ; q_{n_{2}}, x_{2}\right)^{\frac{\alpha_{2}}{2}} K_{n_{2}, p}\left(1 ; q_{n_{2}}, x_{2}\right)^{\frac{2-\alpha_{2}}{2}} \\
\text { we have } & & \ldots \times K_{n_{m}, p}\left(\left(t_{m}-x_{m}\right)^{2} ; q_{n_{m}}, x_{m}\right)^{\frac{\alpha_{m}}{2}} K_{n_{m}, p}\left(1 ; q_{n_{m}}, x_{m}\right)^{\frac{2-\alpha_{m}}{2}} \\
& \mid K_{n_{1}, n_{2}, \ldots, n_{m}, p}\left(f ; q_{n_{1}}, q_{n_{2}}, \ldots, q_{n_{m}}, x_{1}, x_{2}, \ldots, x_{m}\right) & & \leq M \delta_{n_{1}}^{\frac{\alpha_{1}}{2}}\left(x_{1}\right) \delta_{n_{2}}^{\frac{\alpha_{2}}{2}}\left(x_{2}\right) \ldots \delta_{n_{m}}^{\frac{\alpha_{m}}{2}}\left(x_{m}\right) . \\
& -f\left(x_{1}, x_{2}, \ldots, x_{m}\right) \mid & &
\end{array}
$$


Hence, the proof is completed.

\section{Acknowledgements}

The author(s) are very grateful to Prof. P. N. Agrawal, IIT Roorkee, India for his timely help and giving constructive suggestions during the preparation of this paper. Author(s) would also wish to express his gratitude to his parents for their moral support.

\section{References}

[1] P. N. Agrawal, Z. Finta, A. Sathish Kumar: Bernstein-Schurer-Kantorovich operators based on q-integers. Appl. Math. Comput. (submitted).

[2] G. A. Anastassiou and S. Gal, Approximation Theory: Moduli of Continuity and Global Smoothness Preservation, Birkh $\ddot{a}$ user, Boston (2000).

[3] D. Barbosu, Some generalized bivariate Bernstein operators, Math. Notes. 1 (2000) 3-10.

[4] Ö. Dalmanoglu, Approximation by Kantorovich type $q$-Bernstein operators, in: Proceedings of the 12th WSEAS International Conference on Applied Mathematics, Cairo, Egypt, 2007, pp. 113-117, ISSN: 1790-2769.

[5] O. Doğru and V. Gupta, Korovkin-type approximation properties of bivariate $q$-Meyer-König and Zeller operators. Calcolo. 43 (1) (2006) 51-63.

[6] E. Erkuş and O. Duman, A-statistical extension of the Korovkin type approximation theorem. Proc. Indian Acad. Sci. Math. Sci. 115 (4) (2005) 499-507.

[7] S. Ersan and O. Do g ru, Statistical approximation properties of $q$-Bleimann, Butzer and Hahn operators. Math. Comput. Modelling. 49 (2009) 1595-1606.

[8] V. Gupta, C. Radu, Statistical approximation properties of q-Baskokov Kantorovich operators, Cent. Eur. J. Math. 7 (4) (2009) 809818.

[9] V. Kac and P. Cheung, Quantum Calculus, Universitext. Springer, New York 2002.

[10] N.I. Mahmudov, Statistical approximation properties of Baskokov and Baskokov Kantorovich operators based on the q-integers, Cent. Eur. J. Math. 8 (4) (2010) 816826.

[11] N. I. Mahmudov, The moments for $q$-Bernstein operators in the case of $0<q<1$, Numer. Algor., 53 (2010) 439-450.

[12] S. Marinković, P. Rajković and M. Stanković, The inequalities for some types of q-integrals, Comput. Math. Appl. 56 (10) (2008) 2490-2498.

[13] C. V. Muraru, Note on q-Bernstein-Schurer operators. Stud. Univ. Babes-Bolyai Math. 56 (2) (2011) 489-495.

[14] M. Örkcü, Approximation properties of bivariate extension of $q$-Szász-Mirakjan-Kantorovich operators, J. Inequal. Appl. 2013, 2013:324.

[15] M. Örkcü and O. Do g ru, Statistical approximation of a kind of Kantorovich type $q$-Szász-Mirakjan operators. Nonlinear Anal. 75 (5) (2012) 2874-2882.

[16] G. M. Phillips, Bernstein polynomials based on the $q$-integers. Ann. Numer. Math. 4 (1997) 511-518.

[17] C. Radu, Statistical approximation properties of Kantorovich operators based on $q$-integers, Creat. Math. Inform. 17 (2) (2008) 75-84.

[18] F. Schurer, Linear Positive Operators in Approximation Theory, Math. Inst. Tech. Univ. Delft Report., 1962.

[19] D. D. Stancu, A new class of uniform approximating polynomial operators in two and several variables. In: Alexits, G, Stechkin, SB (eds.) Proceedings of the Conference on Constructive Theory of Functions, pp 443-455. Akad é miai, Kiad ó, Budapest (1972).

[20] Marinkovic, S., Rajkovic, P., Stankovic, M.: The inequalities for some types of q-integrals. Comput. Math. Appl. 56(10), 24902498 (2008) 